\title{
РОБАСТНОЕ УПРАВЛЕНИЕ ЗАПАСАМИ В СЕТИ ПОСТАВОК ДЛЯ ПРОИЗВОДСТВА СРЕДСТВ БЫТОВОЙ ХИМИИ
}

\section{Дорофеев Ю.И.}

Национальный технический университет «Харьковский политехнический институт», г. Харьков, e-mail: dorofeev@kpi.kharkiv.edu

Copyright (C) 2014 by author and the journal "Automation technological and business - processes".

This work is licensed under the Creative Commons Attribution International License (CC BY).

http://creativecommons.org/licenses/by/4.0/

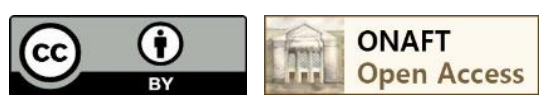

DOI: $10.15673 / 2312-3125.21 /$

\begin{abstract}
Аннотация
Решена задача синтеза робастного стабилизирующего управления запасами в условиях действия неизвестного, но ограниченного внешнего спроса в сети поставок для производства средств бытовой химии ООО Компании «СВ». Параметрическая неоределенность модели, связанная с варьированием значений транспортных запаздываний в процессе функционирования сети поставок, представлена в виде выпуклого многогранника, вершины которого соответствуют возможным вариантам реализации матрицы динамики модели. Для подавления влияния возмущений, моделирующих внешний спрос, одновременно с обеспечением робастной устойчивости замкнутой системы применена методика инвариантных эллипсоидов, которая позволила сформулировать задачу в терминах линейных матричных неравенств, а синтез управления свести к последовательности задач полуопределенного программирования. Приведены результаты численного моделирования.
\end{abstract}

\section{Abstract}

The problem of robust stabilizing inventory control synthesis under the action of an unknown but bounded external demand in the supply network for the production of household chemicals of Ltd. Company "CB" is solved. The parametric uncertainty of the model associated with varying of the transport delays values in the operation of the supply network is provided in the form of a convex polyhedron whose vertices correspond to the possible embodiments of the model dynamic matrix. In order to suppress the influence of disturbances simulating external demand, while ensuring robust stability of the closed-loop system, is used the invariant ellipsoids technique, which allowed to formulate the control problem in terms of linear matrix inequalities. As a result the control synthesis problem is reduced to a sequence of semidefinite programming problems. The results of numerical modeling are presented.

\section{Ключевые слова}

Сеть поставок, управление запасами, робастное управление, метод инвариантных эллипсоидов, линейное матричное неравенство, задача полуопределенного программирования. 


\section{1 ПИТАННЯ ТЕОРІї, МЕТОДИ ТА АЛГОРИТМИ ЕФЕКТИВНОГО АВТОМАТИЧНОГО УПРАВЛІННЯ ОБ’'СКТАМИ ХІМІКО-ТЕХНОЛОГІЧНОГО ТИПУ}

производство, хранение, транспортировку и распределение некоторой продукции с целью удовлетворения потребительского спроса и получения прибыли [1]. Существуют различные типы топологии рассматриваемых систем, которые определяются взаимным размещением производственных звеньев, промежуточных складов и потребителей. Термин «цепь поставок» традиционно используют в случае линейной структуры (конвейера). Если же некоторые виды сырья или полуфабрикатов используются в нескольких процессах, проходящих одновременно, система приобретает эшелонированную структуру. Системы такого типа и являются объектом исследования в данной работе.

Для анализа сетей поставок используется понятие потока. Формально, поток представляет деятельность, которая в единицу времени потребляет некоторые объемы ресурсов, полученные от поставщиков, возможно перерабатывая их, и поставляет определенный объем продукции потребителю. Поток обеспечивает представление в виде «черного ящика» любой производственной деятельности, и не требуют описания, как такая деятельность выполняется. Как частный случай, потоки могут представлять операции транспортировки, перемещающие ресурсы между различными узлами сети без изменения их физических свойств. Отдельно рассматриваются потоки, которые поступают от (или направлены к) внешней среды и представляют внешний спрос либо поставки конечной продукции внешним потребителям. Таким образом, все потоки сети делят на две категории: управляемые (производственные и транспортные операции) и неуправляемые (внешний спрос).

Задача эффективного управления сетями поставок является актуальной для любого предприятия - независимо от его профиля, национальной или территориальной принадлежности и действующей экономической модели. Указанная задача заключается в организации, планировании и управлении информационными, материальными и денежными потоками в сети.

Система управления запасами является важнейшей составляющей системы управления сетями поставок, которая занимается планированием запасов и поставок с учетом выбранной стратегии управления запасами. Под стратегией управления запасами понимается структура правил определения моментов и объемов заказа на восполнение запасов. Оптимальная стратегия должна обеспечивать полное и своевременное удовлетворение как внешнего, так и внутреннего спроса при условии оптимизации некоторого критерия качества работы системы.

Пополнение запасов в сетях поставок всегда происходит с некоторой задержкой относительно момента формирования заказа. Задержки возникают вследствие затрат времени на транспортировку ресурсов между узлами сети, наличия технологических ограничений системы коммуникаций, затрат времени на переработку сырья и полуфабрикатов в узлах сети, наличия человеческого фактора. Сети поставок с запаздываниями различной природы изучались, например, в работе [2].

Существенное влияние на формулировку задач управления запасами оказывают ограничения, которые могут иметь различный характер. Типичными для сетей поставок являются следующие ограничения: 1) значения уровней запаса ресурсов в узлах сети, а также величины управляемых и неуправляемых потоков должны быть неотрицательными; 2) уровни запаса ресурсов, имеющиеся в наличии, не должны превышать максимальные вместимости соответствующих хранилищ; 3) объемы заказов не должны превышать максимально возможные объемы транспортировок.

Таким образом, с точки зрения теории автоматического управления сеть поставок представляет собой многосвязный динамический объект управления, подверженный действию внешних возмущений, роль которых выполняет спрос на ресурсы со стороны внешних потребителей. Возникает необходимость в разработке методов математического моделирования сетей поставок как основы для решения задач управления запасами с целью построения оптимальных в некотором смысле стратегий управления запасами с учетом различных факторов неопределенности и заданных структурных ограничений.

\section{Анализ литературы}

Анализ различных подходов к управлению запасами в сетях поставок можно найти в работе [3] и обширной библиографии к ней. Из всего многообразия методов и моделей управления запасами на практике применяется ограниченное число моделей - в основном те, которые позволяют получить относительно простые способы определения параметров заказа и уровней запасов на складе, а также не требуют сложных методов контроля. Среди таких моделей выделяют два основных типа [1]: модель критических уровней и модель периодической проверки. В первом случае предполагается непрерывный контроль за состоянием запасов и размещение заказов фиксированного размера при снижении текущих запасов до некоторых критических уровней. Второй тип модели предполагает проверку уровня запасов через равные промежутки времени и размещение заказа, размер которого определяется в соответствии с выбранной стратегией. В данной работе рассматривается модель периодической проверки. 


\section{1 ПИТАННЯ ТЕОРІї, МЕТОДИ ТА АЛГОРИТМИ ЕФЕКТИВНОГО АВТОМАТИЧНОГО УПРАВЛІННЯ ОБ'ЄКТАМИ ХІМІКО-ТЕХНОЛОГІЧНОГО ТИПУ}

Выбор стратегии управления запасами определяется характером спроса со стороны внешних потребителей. В настоящее время для синтеза стратегии управления запасами с заданной моделью спроса широко применяется метод прогнозирующего управления [4]. Однако, на практике, как правило, отсутствует информация для построения адекватной модели внешнего спроса, которая необходима для синтеза прогнозирующего управления. Одним из подходов к решению задачи управления запасами в условиях неопределенности спроса является концепция «неизвестных, но ограниченных» воздействий [5]. При этом соответствующая модель спроса характеризуется интервальной неопределенностью.

Если о внешнем спросе, действующем на узлы сети поставок, известно лишь то, что его объемы являются ограниченными, то задача управления запасами может быть сформулирована как задача подавления влияния неслучайных ограниченных внешних возмущений, которая является одной из основных в теории автоматического управления. Одним из подходов к данной проблематике в теории робастного управления является концепция инвариантных множеств, которой посвящена монография [6]. Среди различных форм инвариантных множеств особо выделяются эллипсоиды вследствие их простой структуры и прямой связи с квадратичными функциями Ляпунова.

В рамках метода инвариантных эллипсоидов [7] в качестве технического средства используется математический аппарат линейных матричных неравенств (ЛМН) [8]. После того, как были развиты вычислительные методы, основанные на идеях выпуклой оптимизации, и для их реализации были разработаны соответствующие алгоритмы и программное обеспечение [9], техника ЛМН может рассматриваться в качестве общего метода анализа и синтеза динамических систем при наличии ограничений. При этом, как правило, рассматривают ограничения на ресурс управления, заданные в какой-либо норме. Тогда как спецификой задач управления запасами является неотрицательность значений переменных, что приводит к необходимости учета несимметричных ограничений как на значения управляющих воздействий, так и значения состояний (выходов) сети поставок.

Постановка задачи

Объектом управления является линия по производству чистящего средства TM «SAMA» 500 гр., которое выпускается ООО Компанией «СВ» - ведущим производителем бытовой химии в Украине (http://www.svopter.com.ua).

Состав средства: абразив 90\%, карбонат натрия (сода) 5\%, анионактивные поверхностно активные вещества (ПАВ) менее 5\%, ароматизатор 1\%. Технологический процесс предусматривает изготовление 1 тонны полуфабриката путем смешивания в соответствующих пропорциях абразива, соды и ПАВ, после чего в средство добавляют ароматизатор, расфасовывают в банки по 500 грамм и закрывают крышкой.

Обычно сеть поставок представляют в виде ориентированного взвешенного по дугам графа, вершины которого соответствуют узлам сети и определяют виды и объемы управляемых запасов, а дуги представляют управляемые и неуправляемые потоки в сети. Вес дуги равен времени транспортировки ресурсов между соответствующими узлами сети. Управляемые потоки описывают процессы переработки и перераспределения ресурсов между узлами и процессы поставок сырья извне. Неуправляемые потоки описывают внешний спрос.

Для наглядности вершины графа делят на уровни в зависимости от стадий переработки сырья и полуфабрикатов. Первый уровень содержит вершины, соответствующие узлам сети, на которые действует внешний спрос. Модель соответствующей сети поставок представлена на рис. 1.

Представим управляемые потоки $u_{1}, u_{2}$ и $u_{3}$, описывающие процессы перемешивания ресурсов и расфасовки, в виде гипердуг, а также добавим потоки $u_{4}-u_{10}$, которые описывают поставки сырья извне. Дуги $d_{1}$ и $d_{2}$, изображенные пунктирными линиями, представляют внешний спрос. Значение времени транспортировки $T_{i, j}$ и количество единиц продукции (либо ее вес в тоннах) $\Pi_{i, j}$, которое требуется в соответствии с технологическим процессом для производства 1 тонны конечной продукции, указаны для каждого потока в круглых и квадратных скобках, соответственно. Возле каждого узла в круглых скобках указано значение времени выполнения заказа $T_{i}$. 


\section{I ПИТАННЯ ТЕОРІЇ, МЕТОДИ ТА АЛГОРИТМИ ЕФЕКТИВНОГО АВТОМАТИЧНОГО} УПРАВЛІННЯ ОБ’'СКТАМИ ХІМІКО-ТЕХНОЛОГІЧНОГО ТИПУ

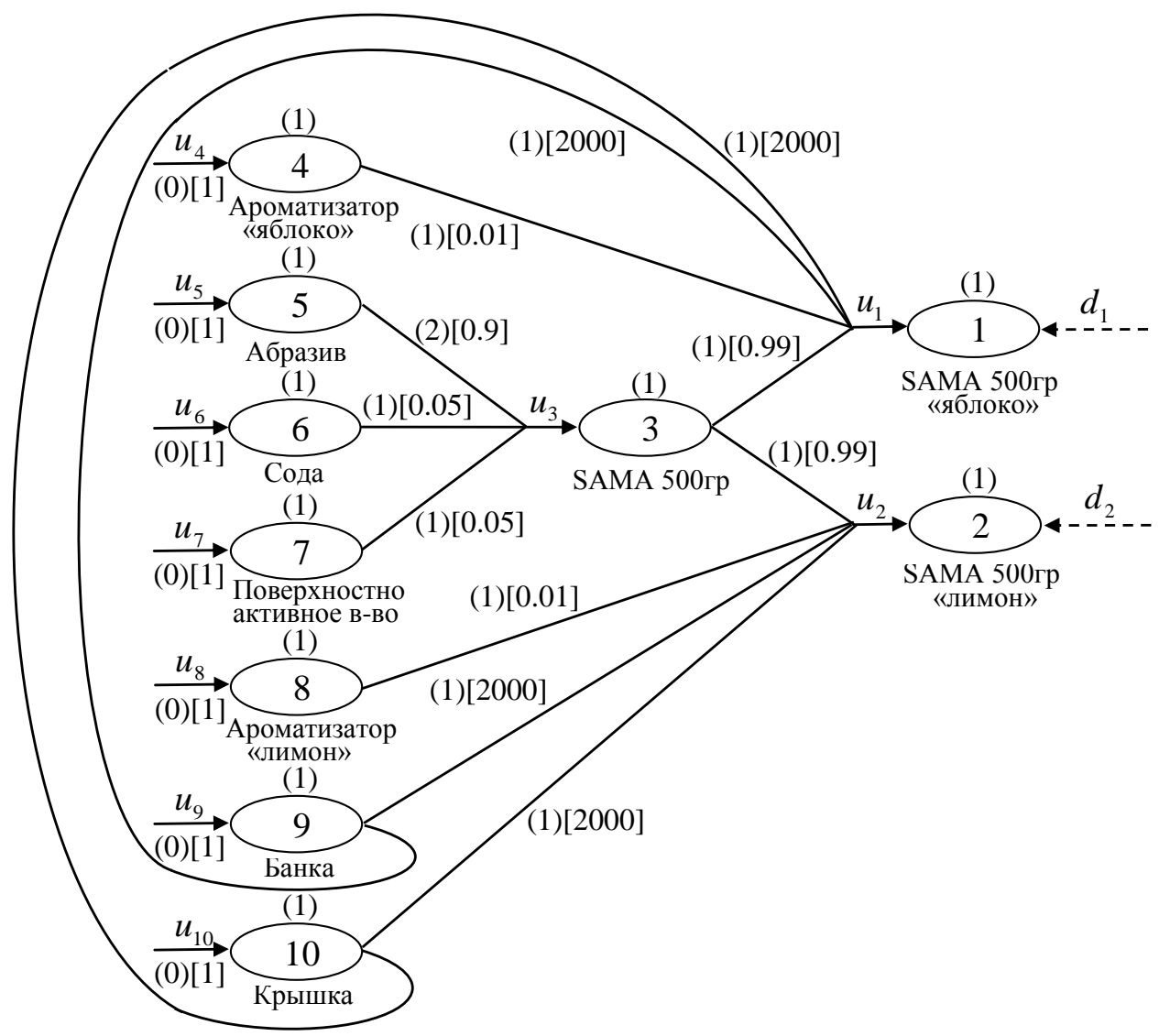

Рис. 1 - Графическое представление модели сети поставок

Для математического описания управляемой сети поставок используется дискретная модель в пространстве состояний, поскольку предполагается, что получение информации о состоянии сети и формирование управляющих воздействий происходит с периодом дискретизации $\Delta t=1$ сутки. Уравнения модели описывают изменение уровня запасов каждого вида ресурсов с течением времени. В качестве переменных состояний рассматриваются наличные уровни запаса ресурсов. Управляющими воздействиями являются объемы заявок на поставку ресурсов, формируемые узлами сети в текущем периоде, а внешними возмущениями выступают объемы спроса на конечную продукцию, которые поступают извне.

Предполагается, что состояния сети поставок доступны непосредственному измерению. Для описания транспортных запаздываний используется модель дискретной задержки. Предполагается, что значения временных интервалов, определяющих длительность транспортировки и переработки ресурсов в узлах сети, известны и кратны выбранному периоду дискретизации. По формуле $\Lambda_{i}=\max \left\{T_{j, i}+T_{i}, i, j=1,10, j \neq i\right\}$ определим величины запаздывания материальных потоков для всех узлов сети. В результате получим, что максимальное значение величины запаздывания управляемых потоков в сети равно 3. Тогда математическая модель управляемой сети поставок задается разностным уравнением с запаздыванием

$$
x(k+1)=x(k)+\sum_{t=0}^{3} B_{t} u(k-t)+E d(k),
$$

где $k=0,1, \ldots$ - номер дискретного интервала; $x(k) \in \boldsymbol{R}^{10}-$ вектор состояний; $u(k) \in \boldsymbol{R}^{10}-$ вектор управляющих воздействий; $d(k) \in \boldsymbol{R}^{2}-$ вектор внешних возмущений.

Начальные условия заданы $x(0)=[2.5,6.0,8.0,0.02,8.0,0.3,0.3,0.04,10000,10000]^{\mathrm{T}}$. Методика построения матриц влияния управлений $B_{t} \in \boldsymbol{R}^{10 \times 10}, t=\overline{0,3}$ и матрицы влияния возмущений $E \in \boldsymbol{R}^{10 \times 2}$ изложена в [10].

В процессе функционирования системы должны выполняться ограничения 


\section{1 ПИТАННЯ ТЕОРІЇ,, МЕТОДИ ТА АЛГОРИТМИ ЕФЕКТИВНОГО АВТОМАТИЧНОГО УПРАВЛІННЯ ОБ'ЄКТАМИ ХІМІКО-ТЕХНОЛОГІЧНОГО ТИПУ}

$$
x(k) \in X=\left\{x \in \boldsymbol{R}^{10}: 0 \leq x \leq x^{\max }\right\}, u(k) \in U=\left\{u \in \boldsymbol{R}^{10}: 0 \leq u \leq u^{\max }\right\},
$$

где векторы $x^{\max }=[3.5,8.0,13.5,0.2,8.5,0.5,0.5,0.2,27000,27000]^{\mathrm{T}}$ и $u^{\max }=[1.5,3.5,6.0,0.05,5.5,0.35,0.35$, $0.05,14000,14000]^{\mathrm{T}}$, определяющие максимальные вместимости хранилищ узлов сети и максимальные объемы транспортировок, заданы.

Для определения граничных значений внешнего спроса использованы данные об объемах спроса на продукцию TM «SAMA» за март 2015 года. Будем предполагать, что векторы, описывающие внешний спрос, удовлетворяют ограничениям

$$
d(k) \in D=\left\{d \in \boldsymbol{R}^{2}: 0 \leq d^{\min } \leq d \leq d^{\max }\right\}
$$

где векторы $d^{\min }=[0,0]^{\mathrm{T}}$ и $d^{\max }=[1.52,3.75]^{\mathrm{T}}$ известны.

Для системы (1) рассматривается задача синтеза автоматического регулятора, который для заданного начального состояния $x(0)$ и неизвестного, но ограниченного спроса $d(k) \in D \forall k \geq 0$ обеспечивает:

1) полное и своевременное удовлетворение как внешнего, так и внутреннего спроса на ресурсы;

2) оптимизацию некоторого критерия качества работы системы;

3) робастную устойчивость замкнутой системы относительно возможных вариаций значений транспортных запаздываний при ограничениях (2).

\section{Синтез робастного стабилизирующего управления}

Первым этапом решения задачи синтеза регулятора является преобразование модели (1) к стандартному виду без запаздывания на основе расширения вектора состояний [11]. Вектор состояний расширенной модели будет равен $\xi(k)=\left[x^{\mathrm{T}}(k), u^{\mathrm{T}}(k-1), u^{\mathrm{T}}(k-2), u^{\mathrm{T}}(k-3)\right]^{\mathrm{T}}$, а уравнения расширенной модели сети примут вид

$$
\begin{aligned}
& \xi(k+1)=A \xi(k)+B u(k)+G d(k), \\
& x(k)=C \xi(k),
\end{aligned}
$$

где матрицы имеют соответствующую блочную структуру

$$
A=\left[\begin{array}{cccc}
I_{10} & B_{1} & B_{2} & B_{3} \\
Z_{10 \times 10} & Z_{10 \times 10} & Z_{10 \times 10} & Z_{10 \times 10} \\
Z_{10 \times 10} & I_{10} & Z_{10 \times 10} & Z_{10 \times 10} \\
Z_{10 \times 10} & Z_{10 \times 10} & I_{10} & Z_{10 \times 10}
\end{array}\right], B=\left[\begin{array}{c}
B_{0} \\
I_{10} \\
Z_{10 \times 10} \\
Z_{10 \times 10}
\end{array}\right], G=\left[\begin{array}{c}
E \\
Z_{10 \times 10} \\
Z_{10 \times 10} \\
Z_{10 \times 10}
\end{array}\right], C^{\mathrm{T}}=\left[\begin{array}{c}
I_{10} \\
Z_{10 \times 10} \\
Z_{10 \times 10} \\
Z_{10 \times 10}
\end{array}\right],
$$

где $I_{10}, Z_{10 \times 10}$ - единичная и нулевая матрицы соответствующих размерностей.

Рассмотрим построение матрицы динамики $A$ расширенной модели сети в том случае, когда величины транспортных запаздываний отличаются от своих номинальных значений. От того, какое значение примет величина запаздывания управляемых потоков $i$-го узла сети зависит, в какой из матриц влияния управляющих воздействий $B_{t}$ элемент $(i, i)$ будет равен 1. Учитывая, что матрицы $B_{t}$ в качестве отдельных блоков входят в состав матрицы динамики, последняя становится нестационарной $A(k)$ и может быть представлена в виде суммы $A(k)=A_{0}+\sum_{i=1}^{3} \lambda_{i}(k) A_{i}$, где набор целых чисел $\lambda_{i}(k), i=\overline{1,3}$ описывает структуру неопределенности и удовлетворяет следующим условиям: $\lambda_{i}(k) \geq 0, \sum_{i=1}^{3} \lambda_{i}(k)=1$. Это означает, что в каждый момент времени $k$ матрица $A(k)$ принимает одно значение из всех возможных вариантов ее реализации, и следовательно, только одно из чисел $\lambda_{i}(k)$ принимает значение 1 , а остальные равны нулю, причем последовательность изменения значений заранее неизвестна. Такой способ описания неопределенности в виде выпуклого многогранника, заданного списком вершин, широко используется в современной теории робастного управления. Учитывая тот факт, что аналогичным образом могут изменяться величины транспортных запаздываний управляемых потоков всех узлов сети, расширенная модель сети поставок в условиях неопределенных значений запаздывания может быть представлена в виде

$$
\begin{aligned}
& \xi(k+1)=A(k) \xi(k)+B u(k)+G d(k), \\
& x(k)=C \xi(k), \quad A(k) \in \Omega=\operatorname{Co}\left\{A^{(1)}, \ldots, A^{(L)}\right\},
\end{aligned}
$$




\section{I ПИТАННЯ ТЕОРІЇ, МЕТОДИ ТА АЛГОРИТМИ ЕФЕКТИВНОГО АВТОМАТИЧНОГО УПРАВЛІННЯ ОБ'ЄКТАМИ ХІМІКО-ТЕХНОЛОГІЧНОГО ТИПУ}

где Co $\{\cdot\}$ - выпуклая оболочка; $A^{(j)}, j=\overline{1, L}-j$-я вершина выпуклого множества $\Omega ; L-$ мощность множества $\Omega$ значений матриц динамики модели при различных значениях интервалов запаздывания управляемых потоков сети.

Будем строить закон управления в виде линейной нестационарной обратной связи по сигналу рассогласования между наличным и страховым уровнями запаса

$$
u(k)=K(k)\left(\xi(k)-\xi^{*}\right)
$$

где $K(k) \in \boldsymbol{R}^{10 \times 40}$ - нестационарная матрица регулятора; $\xi^{*}=\left[x^{* \mathrm{~T}}, x^{* \mathrm{~T}}, x^{* \mathrm{~T}}, x^{* \mathrm{~T}}\right]^{\mathrm{T}}-$ составной вектор, в котором элементы вектора $x^{*}$ определяют размеры страховых запасов ресурсов в узлах сети и вычисляются на основании верхних граничных значений спроса с учетом запаздываний управляемых потоков и продуктивной модели Леонтьева [12]

$$
x^{*}=\left(I_{10}-\ddot{I}\right)^{-1} \hat{d}, \quad \hat{d}_{i}=\left\{\begin{array}{c}
\Lambda_{i} d_{i}^{\max }, i=\overline{1,2} \\
0, \quad i=\overline{3,10}
\end{array}\right.
$$

где $\Pi \in \boldsymbol{R}^{10 \times 10}$ - продуктивная матрица, значение элемента $\Pi_{i, j}$ которой равно количеству единиц ресурса $i$, необходимого для производства единицы ресурса $j$.

Тогда расширенную модель замкнутой системы для управления (5) представим в виде

$$
\begin{aligned}
& \xi(k+1)=(A(k)+B K(k))\left(\xi(k)-\xi^{*}\right)+A(k) \xi^{*}+G\left(d(k)-d^{*}\right)+G d^{*}, \\
& x(k)=C \xi(k), \quad A(k) \in \Omega=\operatorname{Co}\left\{A^{(1)}, \ldots, A^{(L)}\right\} .
\end{aligned}
$$

Выполним аппроксимацию множества $D$ значений внешнего спроса эллипсоидом наименьшего объема, уравнение которого имеет вид

$$
E\left(d^{*}, P_{d}\right)=\left\{d(k) \in \boldsymbol{R}^{2}:\left(d(k)-d^{*}\right)^{\mathrm{T}} P_{d}^{-1}\left(d(k)-d^{*}\right) \leq 1\right\} .
$$

Матрица эллипсоида $P_{d} \in \boldsymbol{R}^{2 \times 2}$ и вектор $d^{*} \in \boldsymbol{R}^{2}$, определяющий координаты центра, вычисляются в результате решения задачи полуопределенного программирования, аналогично тому, как это сделано в работе [13]. В результате получим $d^{*}=[0.760,1.875]^{\mathrm{T}}, P_{d}=\operatorname{diag}(1.155,7.031)$.

Аналогично выполним аппроксимацию множества допустимых значений $X$ эллипсоидом

$$
E\left(x^{*}, P_{x}\right)=\left\{x \in \boldsymbol{R}^{10}:\left(x(k)-x^{*}\right)^{\mathrm{T}} P_{x}^{-1}\left(x(k)-x^{*}\right) \leq 1\right\},
$$

у которого вектор координат центра совпадает с вектором страховых запасов $x^{*}$, а матрица эллипсоида $P_{x}$ вычисляется на основании вектора граничных значений $x^{\max }$ и для рассматриваемого примера равна $P_{x}=\operatorname{diag}\left(4 \cdot 10^{-4}, 1.563,1.482,2 \cdot 10^{-5}, 4.821,0.012,0.012,3 \cdot 10^{-4}, 30691600,30691600\right)$.

Синтез стабилизирующих алгоритмов управления, как правило, основывается на оценивании верхнего граничного значения критерия качества с помощью функции Ляпунова, построенной на решениях системы. Запишем критерий качества в случае бесконечного горизонта в виде

$$
J_{\infty}(k)=\sum_{k=0}^{\infty}\left(\left(\xi(k)-\xi^{*}\right)^{\mathrm{T}} W_{\xi}\left(\xi(k)-\xi^{*}\right)+u(k)^{\mathrm{T}} W_{u} u(k)\right),
$$

где $W_{\xi} \in \boldsymbol{R}^{40 \times 40}, W_{u} \in \boldsymbol{R}^{10 \times 10}$ - положительно определенные диагональные весовые матрицы.

Первое слагаемое в выражении (10) определяет размеры штрафов за отклонение наличных уровней запаса ресурсов от страховых, второе - стоимость производства и транспортировки ресурсов.

В соответствии с методом инвариантных эллипсоидов их можно рассматривать как характеристику влияния внешних возмущений на траекторию динамической системы. Для оценки степени влияния возмущений $d(k) \in E\left(d^{*}, P_{d}\right)$ на выходы $x(k)$ удобно использовать инвариантные эллипсоиды вида

$$
E\left(\xi^{*}, Q(k)\right)=\left\{\xi \in \boldsymbol{R}^{40}:\left(\xi(k)-\xi^{*}\right)^{\mathrm{T}} Q^{-1}(k)\left(\xi(k)-\xi^{*}\right) \leq 1\right\},
$$




\section{1 ПИТАННЯ ТЕОРЇ̈, МЕТОДИ ТА АЛГОРИТМИ ЕФЕКТИВНОГО АВТОМАТИЧНОГО УПРАВЛІННЯ ОБ'ЄКТАМИ ХІМІКО-ТЕХНОЛОГІЧНОГО ТИПУ}

которые аппроксимируют множества достижимости замкнутой системы (7) при действии возмущений $d(k)$.

Тогда задача синтеза регулятора заключается в вычислении в каждый дискретный момент времени $k$ матрицы $K(k)$ такой, чтобы полученный регулятор стабилизировал замкнутую систему (7) и обеспечивал минимизацию по некоторому критерию эллипсоида (11) при ограничениях (2). Для того, чтобы оптимизационная задача соответствовала условиям задач полуопределенного программирования, выберем в качестве критерия сумму квадратов полуосей эллипсоида, то есть след его матрицы $Q(k)$. Соответствующий результат представлен следующей теоремой.

Теорема. Пусть матрицы $\hat{Q}(k) \succ 0$ и $\hat{Y}(k)$ получены в результате решения следующей задачи полуопределенного программирования

$$
\operatorname{trace}(Q(k)) \rightarrow \min
$$

при ограничениях на матричные переменные $Q(k)=Q^{\mathrm{T}}(k) \in \mathbf{R}^{40 \times 40}, Y(k) \in \boldsymbol{R}^{10 \times 40}$ и скаляр $\alpha(k)$

$$
\alpha(k)>0, \quad Q(k) \succ 0,
$$

$$
\begin{aligned}
& {\left[\begin{array}{ccccccc}
Q(k) & Z_{40 \times 40} & Z_{40 \times 2} & \left(A^{(j)} Q(k)+B Y(k)\right)^{\mathrm{T}} & Z_{40 \times 2} & Q(k) W_{\xi} & Y^{\mathrm{T}}(k) W_{u} \\
Z_{40 \times 40} & Z_{40 \times 40} & Z_{40 \times 2} & \left(A^{(j)}-I_{40}\right)^{\mathrm{T}} & Z_{40 \times 2} & Z_{40 \times 40} & Z_{40 \times 10} \\
Z_{2 \times 40} & Z_{2 \times 40} & Z_{2 \times 2} & G^{\mathrm{T}} & Z_{2 \times 2} & Z_{2 \times 40} & Z_{2 \times 10} \\
A^{(j)} Q(k)+B Y(k) & A^{(j)}-I_{40} & G & Q(k) & G P_{d^{\frac{1}{2}}} & Z_{40 \times 40} & Z_{40 \times 10} \\
Z_{2 \times 40} & Z_{2 \times 40} & Z_{2 \times 2} & P_{d^{2}}^{\frac{1}{2}} G^{\mathrm{T}} & \alpha(k) I_{2} & Z_{2 \times 40} & Z_{2 \times 10} \\
W_{\xi} Q(k) & Z_{40 \times 40} & Z_{40 \times 2} & Z_{40 \times 40} & Z_{40 \times 2} & W_{\xi} & Z_{40 \times 10} \\
W_{u} Y(k) & Z_{10 \times 40} & Z_{10 \times 2} & Z_{10 \times 40} & Z_{10 \times 2} & Z_{10 \times 40} & W_{u}
\end{array}\right] \succeq 0, j=\overline{1, L},} \\
& {\left[\begin{array}{cc}
P_{x} & C Q(k) \\
Q(k) C^{\mathrm{T}} & Q(k)
\end{array}\right] \succeq 0,} \\
& Y(k)\left(\xi(k)-\xi^{*}\right) \succeq 0, \quad\left[\begin{array}{cc}
u^{\max }\left(\xi(k)-\xi^{*}\right)^{+} Y^{\mathrm{T}}(k) & Y(k) \\
Y^{\mathrm{T}}(k) & Q(k)
\end{array}\right] \succeq 0,
\end{aligned}
$$

где « ${ }^{+} »-$ псевдообращение Мура-Пенроуза.

Если задача (12)-(15) имеет решение, то регулятор с матрицей

$$
K(k)=\hat{Y}(k) \hat{Q}^{-1}(k)
$$

стабилизирует систему (4) в момент времени $k$ при ограничениях (2).

Доказательство теоремы аналогично доказательству, приведенному в работе [14].

Совокупность ЛМН (13) гарантирует стабилизацию замкнутой системы при действии возмущений $d(k) \in E\left(d^{*}, P_{d}\right)$ и любом значении матрицы динамики $A(k) \in \Omega$, ЛМН (14) обеспечивает выполнение первого из ограничений (2) на значения состояний, а ЛМН (15) - второго из ограничений (2) на значения управляющих воздействий. Отметим, что именно наличие ЛМН (15) приводит к необходимости использования нестационарной обратной связи, поскольку матрицы неравенств зависят от текущего значения вектора состояний $\xi(k)$. Полученный в результате решения задачи (12)-(15) эллипсоид (11) с матрицей $\hat{Q}(k)$ будет инвариантным, то есть фазовая траектория замкнутой системы будет оставаться внутри эллипсоида для всех моментов времени. Это следует из того, что квадратичная форма $\left(\xi(k)-\xi^{*}\right)^{\mathrm{T}} \hat{Q}^{-1}(k)\left(\xi(k)-\xi^{*}\right)$ является функцией Ляпунова для замкнутой системы (7) [15].

\section{Результаты моделирования}

Спецификой рассматриваемой сети поставок является то, что все виды сырья и комплектующих доставляются в течение одного периода (одних суток) с момента заказа, кроме абразива, срок доставки которого двое суток. Предположим, что время транспортировки банок для расфасовки продукции от узла 9 к узлам 1 и 2 в случае форс- 


\section{1 ПИТАННЯ ТЕОРІЇ, МЕТОДИ ТА АЛГОРИТМИ ЕФЕКТИВНОГО АВТОМАТИЧНОГО УПРАВЛІННЯ ОБ'ЄКТАМИ ХІМІКО-ТЕХНОЛОГІЧНОГО ТИПУ}

мажорных обстоятельств может увеличиваться на один период, т.е. $T_{9,1}=T_{9,2}=\{1,2\}$. Тогда величины запаздывания управляемых потоков узлов 1 и 2 могут принимать значения из множества $\Lambda_{1}=\Lambda_{2}=\{2,3\}$. В результате получим $A(k) \in \Omega=\operatorname{Co}\left\{A^{(1)}, A^{(2)}\right\}$, где

$$
A^{(1)}=\left[\begin{array}{cccc}
I_{10} & B_{1} & B_{2}^{(1)} & B_{3}^{(1)} \\
Z_{10 \times 10} & Z_{10 \times 10} & Z_{10 \times 10} & Z_{10 \times 10} \\
Z_{10 \times 10} & I_{10} & Z_{10 \times 10} & Z_{10 \times 10} \\
Z_{10 \times 10} & Z_{10 \times 10} & I_{10} & Z_{10 \times 10}
\end{array}\right], A^{(2)}=\left[\begin{array}{cccc}
I_{10} & B_{1} & B_{2}^{(2)} & B_{3}^{(2)} \\
Z_{10 \times 10} & Z_{10 \times 10} & Z_{10 \times 10} & Z_{10 \times 10} \\
Z_{10 \times 10} & I_{10} & Z_{10 \times 10} & Z_{10 \times 10} \\
Z_{10 \times 10} & Z_{10 \times 10} & I_{10} & Z_{10 \times 10}
\end{array}\right],
$$

а матрица $B_{2}^{(1)}$ отличается от матрицы $B_{2}^{(2)}$ наличием единиц в первых двух строках $\left(\left[B_{2}^{(1)}\right]_{11}=\left[B_{2}^{(1)}\right]_{22}=1\right.$, тогда как $\left.\left[B_{2}^{(2)}\right]_{11}=\left[B_{2}^{(2)}\right]_{22}=0\right)$; аналогично матрица $B_{3}^{(2)}$ отличается от матрицы $B_{3}^{(1)}\left(\left[B_{3}^{(2)}\right]_{11}=\left[B_{3}^{(2)}\right]_{22}=1\right.$, тогда как $\left.\left[B_{3}^{(1)}\right]_{11}=\left[B_{3}^{(1)}\right]_{22}=0\right)$.

Необходимым условием стабилизируемости замкнутой системы является управляемость всех пар матриц $\left(A^{(j)}, B\right), j=\overline{1, L}[11]$. С помощью алгебраического критерия управляемости установлено, что пары матриц $\left(A^{(1)}, B\right)$ и $\left(A^{(2)}, B\right)$ являются управляемыми.

По формуле (6) вычислим уровни страховых запасов узлов сети $x^{*}=[3.04,7.5,10.435,0.030,9.391$, $0.522,0.522,0.075,21080,21080]^{\top}$. Выберем диагональные элементы весовых матриц равными $w_{\xi}=w_{u}=1,0 \cdot 10^{-5}$. Моделирование осуществлялось в течение 22 периодов (количество рабочих дней в марте 2015 года). Вариант реализации матрицы динамики системы $A^{(1)}$ либо $A^{(2)}$ в каждом периоде выбирался случайным образом. Численное решение задачи получено с помощью свободно распространяемого пакета CVX for MATLAB [16]. Результаты моделирования для узлов 1-3 представлены на рис. 2-4, где $a$ - значения граничного, страхового и наличного уровней запасов; $\sigma$ - граничные значения управлений, значения внешнего спроса и управляющих воздействий.

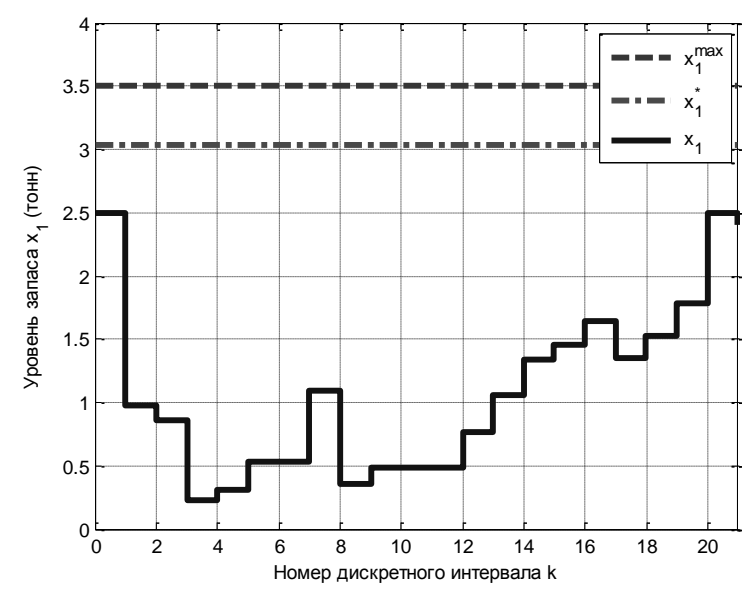

$a$

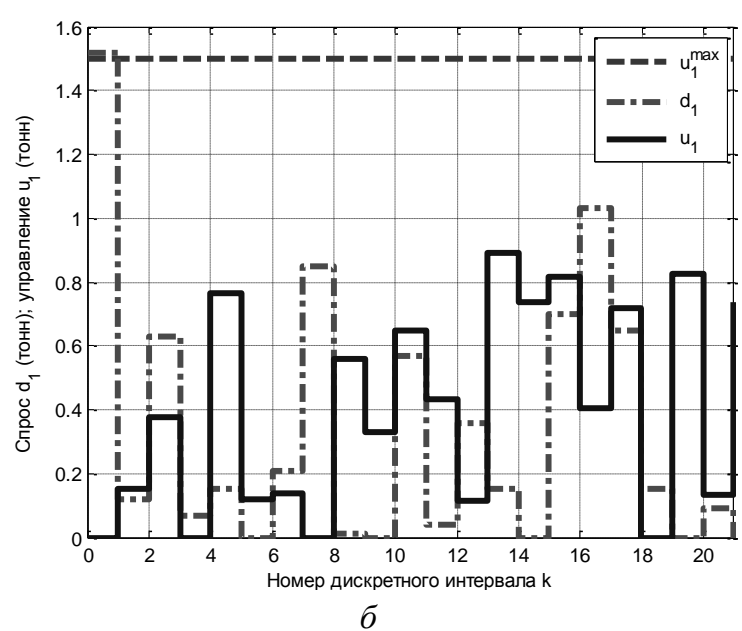

$\sigma$

Рис. 2 - Графики переходных процессов для узла 1 сети поставок 


\section{I ПИТАННЯ ТЕОРІЇ,, МЕТОДИ ТА АЛГОРИТМИ ЕФЕКТИВНОГО АВТОМАТИЧНОГО УПРАВЛІННЯ ОБ'ЄКТАМИ ХІМІКО-ТЕХНОЛОГІЧНОГО ТИПУ}

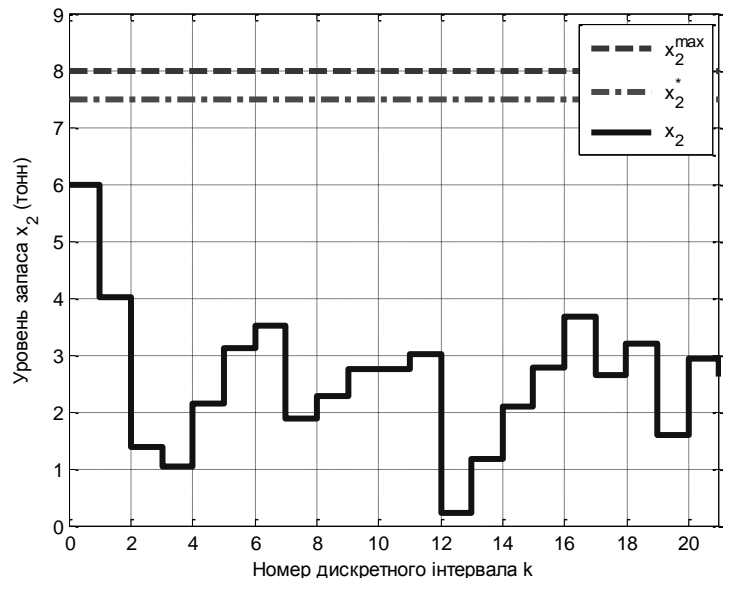

$a$

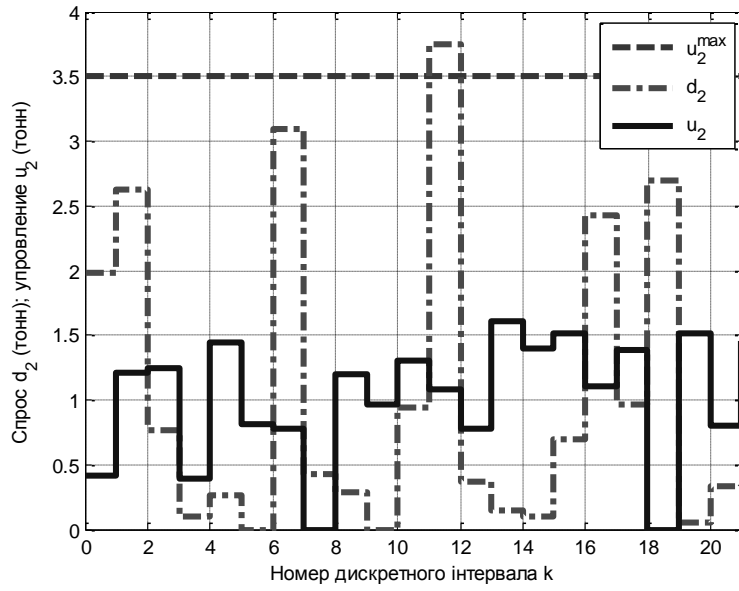

$\sigma$

Рис. 3 - Графики переходных процессов для узла 2 сети поставок

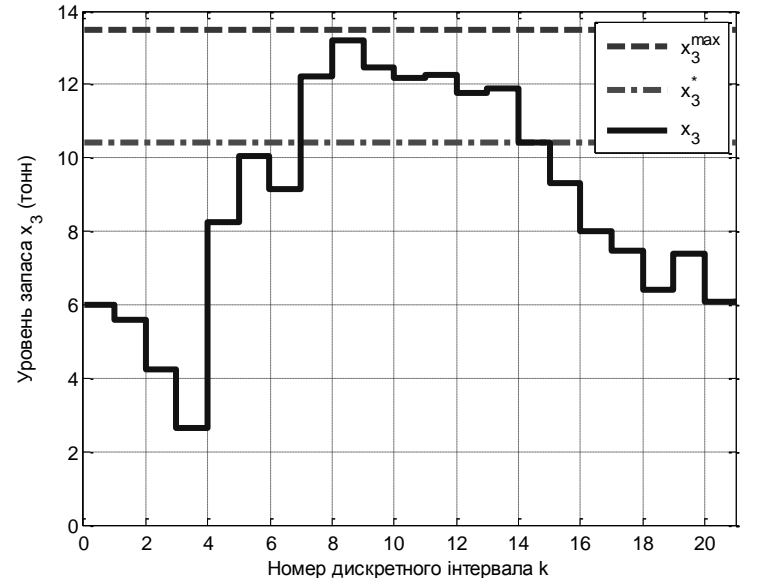

$a$

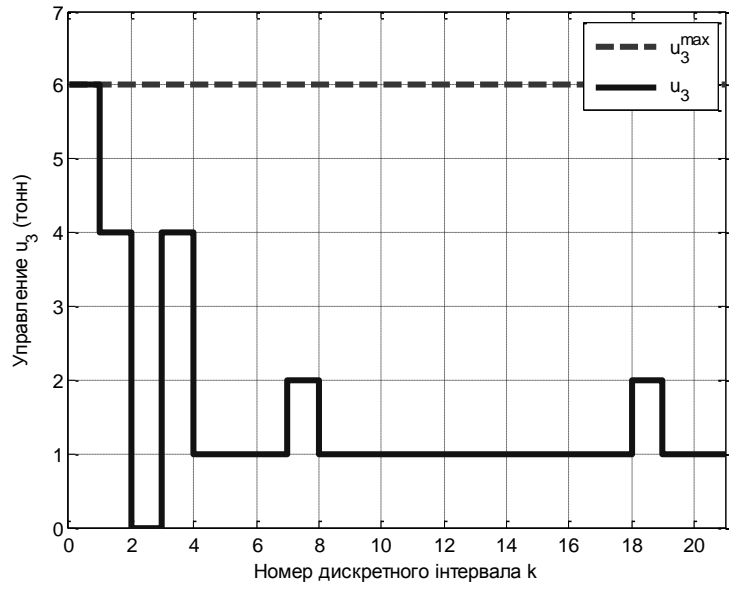

6

Рис. 4 - Графики переходных процессов для узла 3 сети поставок

\section{Выводы}

В работе решена задача синтеза робастного стабилизирующего ограниченного управления запасами в условиях действия неизвестного, но ограниченного внешнего спроса в сети поставок для производства средств бытовой химии ООО Компании «СВ». Для подавления влияния возмущений, моделирующих внешний спрос, одновременно с обеспечением робастной устойчивости замкнутой системы применена методика инвариантных эллипсоидов, которая позволила сформулировать задачу в терминах линейных матричных неравенств, а синтез управления свести к последовательности задач полуопределенного программирования.

Анализ результатов численного моделирования показал, что построенная система управления запасами обеспечивает полное и своевременное удовлетворение как внешнего, так и внутреннего спроса на ресурсы при заданных структурных ограничениях на значения состояний и управляющих воздействий, а также минимизацию затрат, связанных с производством, транспортировкой и хранением ресурсов.

Литература

1. В. А. Лотоцкий и А. С. Мандель, “Модели и методы управления запасами,” М. : Наука, 1991;

2. М. Ю. Киселева и В. И. Смагин, “Управление с прогнозирующей моделью с учетом запаздывания по управлению,” Вестник Томского государственного университета, №2(11), с. 5-12, 2010;

3. А. Н. Стерлигова, “Управление запасами в цепях поставок: учебник,” М. : ИНФРА-М, 2008. 


\section{$\underline{1}$ ПИТАННЯ ТЕОРІЇ, МЕТОДИ ТА АЛГОРИТМИ ЕФЕКТИВНОГО АВТОМАТИЧНОГО УПРАВЛІННЯ ОБ'ЄКТАМИ ХІМІКО-ТЕХНОЛОГІЧНОГО ТИПУ}

4. Е. А. Перепелкин, "Прогнозирующее управление экономической системой производства, хранения и поставок товара потребителям,” Экономика и математические методы, т. 40, № 1, с. 125-128, 2004;

5. D. P. Bertsekas and I. Rhodes, "Recursive state estimation for a set-membership description of uncertainty," IEEE Trans. Autom. Control, vol. 16, pp. 117-128, 1971.;

6. F. Blanchini and S. Miani, "Set Theoretic Methods in Control," Boston: Birkhauser, 2008.;

7. М. В. Хлебников и др., "Оптимизация линейных систем при ограниченных внешних возмущениях (техника инвариантных эллипсоидов),” Автоматика и телемеханика, № 11, с. 9-59, 2011.;

8. S. Boyd et al., "Linear matrix inequalities in system and control theory," Philadelphia: SIAM, 1994.;

9. А.Н. Чурилов и А. В. Гессен, "Исследование линейных матричных неравенств. Путеводитель по программным пакетам,” СПб. : С.-Петерб. гос. ун-т, 2004;

10. Ю. И. Дорофеев и А. А. Никульченко, "Построение математических моделей управляемых сетей поставок с учетом запаздываний потоков,” Системні дослідження та інформаційні технології, № 1, с. 16-27, 2013.;

11. F. Blanchini et al., "Feedback control of production-distribution systems with unknown demand and delays," IEEE Trans. Robot. Autom., vol. 16, no. 3, pp. 313-317, 2000.;

12. А. С. Солодовников и др., “Математика в экономике: учебник,” М. : Финансы и статистика, 1999.;

13. Ю. И. Дорофеев, “Синтез системы оптимального управления запасами с дискретным ПИД-регулятором с использованием техники линейных матричных неравенств," Збірник наукових праць Харківського університету Повітряних Сил, вип. 4(41), с. 34-41, 2014.;

14. Ю. И. Дорофеев и др., "Робастное стабилизирующее управление запасами в сетях поставок в условиях неопределенности внешнего спроса и интервалов задержки пополнения запасов," Известия РАН. Теория и системы управления, № 5, с. 146-160, 2014.;

15. Ю. И. Дорофеев, “Подавление влияния ограниченных внешних возмущений в системе управления запасами цепи поставок," Автоматизация технологических и бизнес-процессов, № 20, с. 113-120, 2015.;

16. M. Grant and S. Boyd, "CVX: Matlab software for disciplined convex programming, version 2.0 beta," http://cvxr.com/cvx (2013).

\section{References}

1. V. A. Lotockij i A. S. Mandel', "Modeli i metody upravlenija zapasami," M. : Nauka, 1991.;

2. M. Yu. Kiseleva i V. I. Smagin, "Upravlenie s prognozirujushhej model'ju s uchetom zapazdyvanija po upravleniju," Vestnik Tomskogo gosudarstvennogo universiteta, №2(11), s. 5-12, 2010.;

3. A. N. Sterligova, "Upravlenie zapasami v cepjah postavok: uchebnik," M. : INFRA-M, 2008.;

4. E. A. Perepelkin, "Prognozirujushhee upravlenie jekonomicheskoj sistemoj proizvodstva, hranenija i postavok tovara potrebiteljam," Jekonomika i matematicheskie metody, t. 40, № 1, s. 125-128, 2004.;

5. D. P. Bertsekas and I. Rhodes, "Recursive state estimation for a set-membership description of uncertainty," IEEE Trans. Autom. Control, vol. 16, pp. 117-128, 1971.;

6. F. Blanchini and S. Miani, "Set Theoretic Methods in Control," Boston: Birkhauser, 2008.;

7. M. V. Hlebnikov i dr., "Optimizacija linejnyh sistem pri ogranichennyh vneshnih vozmushhenijah (tehnika invariantnyh jellipsoidov)," Avtomatika i telemehanika, № 11, s. 9-59, 2011.;

8. S. Boyd et al., "Linear matrix inequalities in system and control theory," Philadelphia: SIAM, 1994.;

9. A. N. Churilov i A. B. Gessen, "Issledovanie linejnyh matrichnyh neravenstv. Putevoditel' po programmnym paketam," SPb. : S.-Peterb. gos. un-t, 2004.;

10. Yu. I. Dorofieiev i A. A. Nikulchenko, "Postroenie matematicheskih modelej upravljaemyh setej postavok s uchetom zapazdyvanij potokov," Sistemni doslidzhennja ta informacijni tehnologiï, № 1, s. 16-27, 2013.;

11. F. Blanchini et al., "Feedback control of production-distribution systems with unknown demand and delays," // IEEE Trans. Robot. Autom., vol. 16, no. 3, pp. 313-317, 2000.;

12. A. S. Solodovnikov i dr., "Matematika v jekonomike: uchebnik," M. : Finansy i statistika, 1999.;

13. Yu. I. Dorofieiev, "Sintez sistemy optimal'nogo upravlenija zapasami s diskretnym PID-reguljatorom s ispol'zovaniem tehniki linejnyh matrichnyh neravenstv," Zbirnik naukovih prac' Harkivs'kogo universitetu Povitrjanih Sil, vip. 4(41), s. 34-41, 2014.;

14. Yu. I. Dorofieiev i dr., "Robastnoe stabilizirujushhee upravlenie zapasami v setjah postavok v uslovijah neopredelennosti vneshnego sprosa i intervalov zaderzhki popolnenija zapasov,” Izvestija RAN. Teorija i sistemy upravlenija, № 5, s. 146-160, 2014.;

15. Yu. I. Dorofieiev, "Podavlenie vlijanija ogranichennyh vneshnih vozmushhenij v sisteme upravlenija zapasami cepi postavok," Avtomatizacija tehnologicheskih i biznes-processov, № 20, s. 113-120, 2015.

16. M. Grant and S. Boyd, "CVX: Matlab software for disciplined convex programming, version 2.0 beta," http://cvxr.com/cvx (2013). 